\title{
Clinical application of a new hemostatic material using mussel-inspired catecholamine hemostat: A pilot study
}

\author{
Young-Mok Park' ${ }^{1}$, Hyung-II Seo', Jae-Hoon Kim², Sung Pil Yoon ${ }^{3}$, Haeshin Lee ${ }^{4}$, Moon Sue Lee ${ }^{5}$ \\ 'Department of Surgery, Biomedical Research Institute, Pusan National University Hospital, \\ Pusan National University School of Medicine, Busan, Korea, \\ ${ }^{2}$ Department of Trauma Surgery and Surgical Critical Care, Biomedical Research Institute, \\ Pusan National University Hospital, Pusan National University School of Medicine, Busan, Korea, \\ ${ }^{3}$ Department of Surgery, Gang-an Hospital, Busan, Korea, \\ ${ }^{4}$ Department of Chemistry, Korea Advanced Institute of Science and Technology (KAIST), Daejeon, Korea, \\ ${ }^{5}$ InnoTherapy Inc., Seoul, Korea
}

Backgrounds/Aims: This study aimed to evaluate clinical application of InnoSEAL Plus (a mussel-inspired catecholamine hemostat) as a new hemostatic material for humans.

Methods: Patients treated with topical hemostatic patches after liver resection were enrolled. They were divided into an experimental group (InnoSEAL Plus group) and two control groups (TachoSil ${ }^{\circledR}$ group and Surgicel Fibrillar ${ }^{\circledR}$ group) for efficacy evaluation.

Results: A total of 15 patients were enrolled. Each group had five patients. The 3 -minute hemostasis success rate was $80.0 \%(4 / 5$ patients) in the InnoSEAL Plus group, 80.0\% (4/5 patients) in the TachoSil ${ }^{\circledR}$ group, and 40.0\% (2/5 patients) in the Surgicel Fibrillar ${ }^{\circledR}$ group, showing no significant difference in the success rate among these groups $(p>0.05)$. All three groups exhibited $100 \%$ success rate for 10-minute hemostasis. Both InnoSEAL Plus and TachoSil ${ }^{\mathbb{B}}$ groups had one patient developing adverse events, which were treated easily with drug administrations.

Conclusions: InnoSEAL Plus is expected to be functionally not inferior to other conventional hemostatic agents. However, it is necessary to confirm this through multicenter prospective studies in the future.

Key Words: Pilot study; Hemostatics; Hepatectomy; Mussel adhesion

\section{INTRODUCTION}

Advances in surgical technique have reduced the operative mortality and morbidity following liver resection. Although the mortality rate has decreased to be $<5 \%$, morbidity rate of up to $40 \%$ is still reported [1]. In addition, postoperative bleed-

Received: May 31, 2021, Revised: August 2, 2021,

Accepted: August 16, 2021

Corresponding author: Hyung-II Seo

Department of Surgery, Biomedical Research Institute, Pusan National University Hospital, Pusan National University School of Medicine, 179 Gudeok-ro, Seo-gu, Busan 49241, Korea

Tel: +82-51-240-7238, Fax: +82-51-247-1365, E-mail: seohi71@hanmail.net ORCID: https://orcid.org/0000-0002-4132-7662

Copyright (C) The Korean Association of Hepato-Biliary-Pancreatic Surgery This is an Open Access article distributed under the terms of the Creative Commons Attribution Non-Commercial License (http://creativecommons.org/licenses/by-nc/4.0) which non-commercial use, distribution, and reproduction in any medium, provided the original permits unrestricted work is properly cited. ing and bile leakage remain challenging complications. They might lead to transfusion and prolonged hospitalization for patients who have undergone a liver resection [2]. Technical advances for the management of these complications include diathermy, argon beam coagulation, suture, clipping, hepatic inflow occlusion, and vascular occlusion. Topical agents including collagen fleeces, bovine collagen-based composite mixed with autologous plasma, and fibrin sealants have also been developed as supporting measures to prevent these problems [3-5].

Topical agents may help reduce postoperative bleeding and bile leak through effective sealing of vessel perforations and biliary radicals. Therefore, these agents are important in hepatic surgery. Recently, numerous mussel-inspired biomaterials have been developed as tissue adhesives, hydrogels for drug depots, hemostats, and surface-functionalizing polymers. In particular, a new class of adhesive hemostats for arresting bleeding has been reported [6-16]. 
Our previous study has used catechol-conjugated chitosan (CHI-C) as a hemostatic material to demonstrate cathechol-cation synergy (hemostatic ability of a CHI-C sponge in a heparinized rabbit model of femoral artery bleeding and a pig model of traumatic blunt liver injury with hemodilutional and hypothermic coagulopathy) [17]. We hypothesized that the use of chitosan-catechol might exhibit a superior medical sealant function focusing on adhesive hemostatic materials and that the sealing function might act as fibrin like functions even if hemostatic materials contains no fibrins. InnoSEAL Plus is the first-kind this type of device without fibrin yet exhibiting equivalent hemostatic capability. Well-designed and accurately executed good research is needed to produce high-quality results. To that end, it is very helpful to review the feasibility of a main study prior to performing it.

Thus, the aim of this pilot study was to recruit research participants and evaluate the feasibility of research of using InnoSEAL Plus (InnoTherapy, Seoul, Korea), a mussel-inspired catecholamine hemostat, for human patients.

\section{PATIENTS AND METHODS}

\section{Patients and study design}

We conducted a single-center, controlled, single-blind, and exploratory clinical study on early hemostatic efficacy and safety of InnoSEAL Plus. To approve clinical trials, all necessary toxicity tests including ISO-10993 (ISO 10993-5, cytotoxicity; ISO 10993-6, histopathological test; ISO 1099310 , intracutanenous reactivity and skin sensitization; and ISO 10993-11, acute toxicity and subchronic toxicity) were passed. The clinical study was designed as a comparative study with TachoSil $^{\circledR}$ (Nycomed, Cophenhagen, Denmark) and Surgicel
Fibrillar $^{\circledR}$ (Ethicon, Somerville, NJ, USA). Adult patients (age $>18$ years) indicated for elective liver resection for any reason (e.g., hepatocellular carcinoma, intrahepatic cholangiocarcinoma, intrahepatic duct stone, and metastatic liver disease) were eligible. Those who had a failure of first-line coagulation therapy (including diathermy, argon beam coagulation, suture, and clipping), liver cirrhosis with portal hypertension or above Child-Pugh B, those who underwent emergency hepatic resection, and those who had hypersensitivity to chito acid were excluded. This pilot study was approved by the Institutional Review Board (IRB) at Pusan National University Hospital Clinical Trial Center (IRB number: 1604-007-040). All patients provided written informed consent prior to study participation.

As a pilot study involving a small number of subjects, patients were assigned to an experimental group (InnoSEAL Plus group) and two control groups (TachoSil ${ }^{\circledR}$ group and Surgicel Fibrillar $^{\circledR}$ group) based on the order of registration to the study instead of using a randomization method (Surgicel Fibrillar ${ }^{\circledR}$ - TachoSil $^{\circledR}$ - InnoSEAL Plus - Surgicel Fibrillar ${ }^{\circledR}$ - TachoSil $^{\circledR}$ - InnoSEAL Plus -...). To evaluate the feasibility of the recruitment of study participants and the study design, not the sample size, to test the hemostatic effect of InnoSEAL Plus, a total of 15 patients (5 patients for each group) were enrolled.

\section{Hemostatic methods}

After liver resection, first-line coagulation therapy, including diathermy, argon beam coagulation, suture, and clipping, was performed. When the surgeon determined that hemostasis was achieved, the liver surface was compressed with a dry gauze for 1 minute for confirmation. If bleeding from exposed vessel was seen, coagulation therapy was repeated to achieve complete

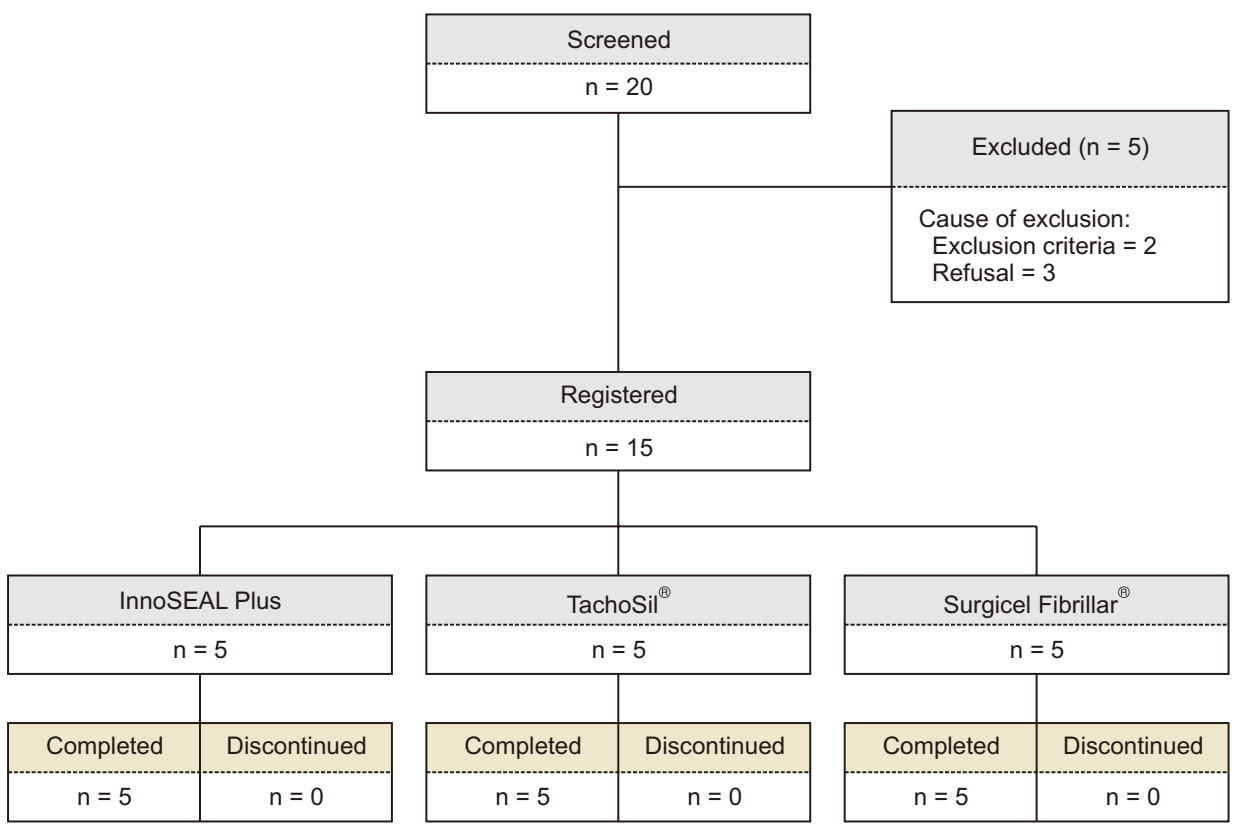

Fig. 1. Flow diagram showing patient enrollment. 
hemostasis. Following this process after controlling active bleeding, each patch product was applied at the liver cut surface. When major liver resection was performed, two patches and a single layer were usually applied. However, the number of patches and layers depended on the severity and the extent of operative lesion. Patches were compressed with a wet gauze for 30 seconds.

\section{Outcome measures}

Effectiveness was evaluated according to the following parameters: time to hemostasis, hemostasis success rate within 3 and 10 minutes, and re-bleeding rate. Time to hemostasis was defined as the time between application of the patch to liver surface up to the time when there was no longer any evidence of bleeding. Hemostasis success rate was defined as the rate of complete hemostasis within 3 or 10 minutes from the application of patch to the liver surface until the time when there was no longer any evidence of bleeding. Re-bleeding rate was defined as the frequency of the re-occurrence of bleeding because of failure of hemostasis after patch application that required any additional treatment.

The safety of InnoSEAL Plus was evaluated throughout the study period. Patients were admitted for at least 7 days after surgery for evaluation. The final evaluation was conducted on the 30th postoperative day. Computed tomography (CT) was used to identify ascites, bilomas, and other abnormal fluid collections at postoperative day 5 .
An adverse event was defined as any undesirable clinical change, whether or not the condition was not detected before surgery. This event was reported using systemic organ class and preferred term under MedDRA (version 19.0; ICH, Geneva, Switzerland).

\section{Statistical analysis}

Interim analysis and subgroup analysis were conducted for the safety and effectiveness of this study. Statistical significance between treatment groups was confirmed using Kruskal-Wallis test. For factors with a $p$-value $<0.05$, post-hoc test was performed using Wilcoxon rank sum test for additional verification. Fisher's exact test was used for categorical data evaluation. For factors with a $p$-value $<0.05$, post-hoc test was performed using Wilcoxon rank sum test for additional verification.

As this study was an exploratory study, adjustment for multiple comparisons was not made. Statistical significance was judged based on a bilateral significance of 0.05 . All statistical analyses were performed using SAS program version 9.3 (SAS Institute, Cary, NC, USA).

\section{RESULTS}

\section{Patient characteristics}

Of 20 patients eligible for this study, five were excluded, leaving 15 patients for the trial. These patients were divided equally

Table 1. Summary of baseline demographic characteristics

\begin{tabular}{|c|c|c|c|c|}
\hline Characteristic & $\begin{array}{c}\text { InnoSEAL Plus } \\
(\mathrm{n}=5)\end{array}$ & $\begin{array}{c}\text { TachoSil }^{\circledR} \\
(n=5)\end{array}$ & $\begin{array}{l}\text { Surgicel Fibrillar }{ }^{\circledR} \\
\qquad(n=5)\end{array}$ & $p$-value \\
\hline Sex (male : female) & $2: 3$ & $4: 1$ & $3: 2$ & 0.8002 \\
\hline Age $(y r)^{a)}$ & $70.60 \pm 6.84$ & $58.80 \pm 9.04$ & $63.20 \pm 6.69$ & 0.1416 \\
\hline Resection type & & & & 0.4172 \\
\hline Major & 1 & 3 & 3 & \\
\hline Minor & 4 & 2 & 2 & \\
\hline Diagnosis & & & & 0.4190 \\
\hline $\mathrm{HCC}$ & 3 & 2 & 3 & \\
\hline Metastasis & 0 & 2 & 1 & \\
\hline Cholangiocarcinoma & 0 & 1 & 0 & \\
\hline Gallbladder cancer & 1 & 0 & 0 & \\
\hline IPBN & 1 & 0 & 0 & \\
\hline IHD stone & 0 & 0 & 1 & \\
\hline Underlying liver disease & & & & 0.6120 \\
\hline Hepatic B & 2 & 3 & 2 & \\
\hline Hepatitis C & 2 & 0 & 1 & \\
\hline Comorbidity & & & & 0.7410 \\
\hline Hypertension & 4 & 3 & 3 & \\
\hline Diabetes & 1 & 2 & 2 & \\
\hline
\end{tabular}

Values are presented as number only or mean \pm standard deviation.

HCC, hepatocellular carcinoma; IPBN, intraductal papillary biliary neoplasm; IHD, intraductal hepatic duct.

${ }^{\text {a) }}$ The mean age of the overall cohort was $64.20 \pm 8.65$ years. 
Table 2. Hemostasis time and success rates at 3 minutes and 10 minutes and re-bleeding rates

\begin{tabular}{|c|c|c|c|}
\hline End point & InnoSEAL Plus $(n=5)$ & TachoSil $^{\mathbb{R}}(n=5)$ & Surgicel Fibrillar ${ }^{\circledR}(n=5)$ \\
\hline \multicolumn{4}{|l|}{ Time to hemostasis (min) } \\
\hline Mean \pm standard deviation & $2.80 \pm 1.10$ & $3.00 \pm 1.87$ & $4.80 \pm 2.59$ \\
\hline Median (range) & $3.00(1.00-4.00)$ & $3.00(1.00-6.00)$ & $4.00(2.00-8.00)$ \\
\hline$p$-value & & 0.9110 & 0.2796 \\
\hline \multicolumn{4}{|l|}{3 minutes } \\
\hline Success (\%) & $4(80.0)$ & $4(80.0)$ & $2(40.0)$ \\
\hline$p$-value & & Not estimated & 0.5238 \\
\hline \multicolumn{4}{|l|}{10 minutes } \\
\hline Success (\%) & $5(100)$ & $5(100)$ & $5(100)$ \\
\hline$p$-value & & Not estimated & Not estimated \\
\hline \multicolumn{4}{|l|}{ Re-bleeding } \\
\hline Yes (\%) & $0(0)$ & $0(0)$ & $0(0)$ \\
\hline No (\%) & $5(100)$ & $5(100)$ & $5(100)$ \\
\hline$p$-value & & Not estimated & Not estimated \\
\hline
\end{tabular}

into the InnoSEAL Plus group $(\mathrm{n}=5)$, the TachoSil ${ }^{\circledR}$ group $(\mathrm{n}=$ 5), and the Surgicel Fibrillar ${ }^{\mathbb{B}}$ group $(n=5)$ (Fig. 1$)$.

The mean age of the overall cohort was $64.20 \pm 8.65$ years. The number of male patients in the InnoSEAL Plus was low at $40 \%$. The mean age was higher in InnoSEAL Plus $(70.60 \pm$ 6.84 years). However, there was no statistical difference in sex distribution or mean age between groups. Major ( $>3$ segments) and minor ( $<3$ segments) resection were performed for 7 and 8 patients, respectively. Causes of liver resection were hepatocellular carcinoma ( $n=8$ cases), metastasis $(n=3)$, intrahepatic cholangiocarcinoma $(n=1)$, gallbladder cancer $(n=1)$, intraductal papillary biliary neoplasm $(n=1)$, and hepatolithiasis ( $n$ $=1$ ). Clinical data of these 15 patients are shown in Table 1.

\section{Analysis of effectiveness}

Regarding hemostatic time, the time to hemostasis in the InnoSEAL Plus group was shorter than that in the TachoSil ${ }^{\mathbb{R}}$ group or the Surgicel Fibrillar ${ }^{\mathbb{B}}$ group $(2.80 \pm 1.10$ minutes vs. $3.00 \pm 1.87$ minutes or $4.80 \pm 2.59$ minutes). However, difference between groups was not statistically significant $(p>0.05)$ (Table 2). Regarding the 3-minute hemostasis success rate, the InnoSEAL Plus group and TachoSil ${ }^{\circledR}$ group both achieved $80.0 \%(4 / 5)$, while it was lower in the Surgicel Fibrillar ${ }^{\circledR}$ group at $40.0 \%(2 / 5)$. However, the 3-minute hemostasis success rate was not significantly different between groups $(p>0.05)$. For the 10 -minute hemostasis success rate, a $100 \%$ rate was achieved for all three groups. No re-bleeding occurred in any of the three groups (Table 2).

\section{Analysis of safety}

A total of 160 cases of adverse events occurred: 62 cases in the InnoSEAL Plus group, 56 cases in the TachoSil ${ }^{\circledR}$ group, and 42 cases in the Surgicel Fibrillar ${ }^{\mathbb{B}}$ group. More than $50 \%$ of patients experienced adverse events of injury, poisoning, procedural complications, hepatobiliary disorders, respiratory, thoracic, and mediastinal disorders, metabolism and nutrition disorders, general disorders and administration site complications, and gastrointestinal disorders. Injury, poisoning, and procedural complications accounted for $80.0 \%$ of all cases of adverse events $(80.0 \%$ in the InnoSEAL Plus group, $100 \%$ in the $\mathrm{TachoSil}^{\circledR}$ group, and $100 \%$ in the Surgicel Fibrillar ${ }^{\circledR}$ group) and hepatobiliary disorders $(80.0 \%$ in the InnoSEAL Plus group, $100 \%$ in the TachoSil ${ }^{\circledR}$ group, and $80.0 \%$ in the Surgicel Fibrillar $^{\circledR}$ group).

Procedural pain, abnormal hepatic function, disorders of respiratory gas exchange, and pyrexia known to be common in liver resection occurred in $>50 \%$ of patients. In terms of serious adverse events, one (20.0\%) patient in the InnoSEAL Plus group developed 7 adverse events (namely body fluid imbalance and peripheral edema, ear edema and external ear pain, and hypotony maculopathy and scleritis) at 4,5 , and 6 days after application of InnoSEAL Plus. In this situation, there were no abnormal findings for allergy reaction test associated with InnoSEAL Plus. All these were treated with drug administration. Meanwhile, one $(20.0 \%)$ patient in the TachoSil ${ }^{\circledR}$ group developed three adverse events. No case of adverse events causing dropout or death occurred during the trial.

All patients underwent abdominal CT. There was no evidence of re-bleeding, bilomas, or abnormal fluid collection. Additional treatment was not required for bile leak or other resectional surface-related complications. Despite a high frequency of adverse events, no serious adverse event caused death study drop out. Moreover, the most frequent adverse events were those commonly occurring in liver resection. Only one patient in the InnoSEAL Plus group developed a serious adverse event. All adverse events were considered to be either unrelated or unlikely to be related to InnoSEAL Plus treatment. All patients recovered without additional treatment or complication. 


\section{DISCUSSION}

Bleeding is a leading cause of intra- and post-operative morbidity and mortality. Although various hemostats have been developed, arresting perioperative bleeding remains a challenge [18]. Effective hemostasis is essential in surgery. No surgery can be finished unless complete hemostasis is accomplished. Advances in biomaterials have led to the discovery of various topical hemostatic agents, which are primarily classified as biologically active and passive hemostatic materials [19,20]. Fibrin glues are the most commonly used active hemostats. They contain physiologically active thrombin and fibrinogen. However, fibrin glues have a latent risk of viral contamination (i.e., HIV and hepatitis A, B, and C) and prion disease transmission [1921]. Importantly, fibrin glues are not effective against massive, high-pressure bleeding due to their poor tissue adhesion and weak cohesive strength [21-23]. Although passive hemostatic materials such as collagen, gelatin, glutaraldehyde-albumin, cyanoacrylate, and oxidized cellulose do not contain active coagulation factors, they can indirectly cause platelet activations and aggregations [24,25]. Previous studies have shown that collagen-, gelatin-, and oxidized cellulose-based hemostats have poor performances and often led to re-bleeding that require reoperations [24,25]. Meanwhile, concerns about significant tissue toxicity from degraded byproducts and exothermal polymerizations have been raised for cyanoacrylates and semi-synthetic-like glutaraldehyde-albumin sealants [19-21].

Numerous mussel-inspired biomaterials such as hyaluronic acid-catechol, multi-armed poly(ethylene glycol)-catechol, dextran-catechol, alginate-catechol, heparin-catechol, and poly(ethylenimine)-catechol have been recently developed [612]. In general, mussel-inspired adhesive biomaterials are multifunctional in that they can be used as tissue adhesives, hydrogels for drug depots, hemostats, and surface-functionalizing polymers. In particular, a new class of adhesive hemostats for arresting bleeding has been reported, including catechol-functionalized chitosan/pluronic hydrogels, poly(organophophazene)-catechol, polyethylene glycol-citric acid-catechol, and gelatin-catechol [13-16]. Unique characteristics of these materials are that they simultaneously show both hemostatic and tissue sealing properties. They show excellent hemostatic capability by strengthening polymer network structures and adhesiveness to tissues. However, whether mussel-inspired catecholamine can arrest bleeding after hepatic resection has never been reported.

In this study, mussel-inspired catecholamine was compared with two different commercial products. Results showed no significant difference in hemostatic time, success rate, or re-bleeding rate between the mussel-inspired catecholamine and commercial products. All data for this pilot study of InnoSEAL Plus were handled by a Ministry of Food and Drug Safety reviewer. The initial hemostasis success time was set as 3 minutes in this study based on previous studies showing a mean hemostatic time of 2.5-4.7 minutes [4,26,27]. In addition, to confirm hemostasis, the operation was terminated after waiting for an additional 10 minutes and verifying no bleeding. There were no significant differences in the hemostatic time between the experimental group and control groups.

This pilot study is a preliminary study conducted to evaluate the validity of the main study to be officially started. Through this study, the authors obtained the basis for performing a full randomized clinical study. In conclusion, this pilot study showed that InnoSEAL Plus, a chitosan-catechol conjugate, might be useful as a hemostatic material for liver resection. Results of this study warrant further multicenter prospective studies.

\section{FUNDING}

None.

\section{CONFLICT OF INTEREST}

No potential conflict of interest relevant to this article was reported.

\section{ORCID}

Young-Mok Park, https://orcid.org/0000-0002-4165-3054

Hyung-Il Seo, https://orcid.org/0000-0002-4132-7662

Jae-Hoon Kim, https://orcid.org/0000-0003-4504-9898

Sung Pil Yoon, https://orcid.org/0000-0002-8910-4249

Haeshin Lee, https://orcid.org/0000-0003-3961-9727

Moon Sue Lee, https://orcid.org/0000-0001-8492-9893

\section{AUTHOR CONTRIBUTIONS}

Conceptualization: HIS. Data curation: HIS, YMP, HL, JHK. Methodology: HIS, YMP, HL, JHK. Visualization: HIS. Writing - original draft: HIS, YMP, HL. Writing - review \& editing: All author.

\section{REFERENCES}

1. Aragon RJ, Solomon NL. Techniques of hepatic resection. J Gastrointest Oncol 2012;3:28-40.

2. Nagano Y, Togo S, Tanaka K, Masui H, Endo I, Sekido H, et al. Risk factors and management of bile leakage after hepatic resection. World J Surg 2003;27:695-698.

3. Morgenstern L, Michel SL, Austin E. Control of hepatic bleeding with microfibrillar collagen. Arch Surg 1977;112:941-943.

4. Chapman WC, Clavien PA, Fung J, Khanna A, Bonham A. Effective control of hepatic bleeding with a novel collagen-based composite combined with autologous plasma: results of a randomized controlled trial. Arch Surg 2000;135:1200-1204.

5. Jackson MR, MacPhee MJ, Drohan WN, Alving BM. Fibrin sealant: 
current and potential clinical applications. Blood Coagul Fibrinolysis 1996;7:737-746.

6. Hong S, Yang K, Kang B, Lee C, Song IT, Byun E, et al. Hyaluronic acid catechol: a biopolymer exhibiting a $\mathrm{pH}$-dependent adhesive or cohesive property for human neural stem cell engineering. Adv Funct Mater 2013;23:1774-1780.

7. Lee BP, Dalsin JL, Messersmith PB. Synthesis and gelation of DOPA-modified poly(ethylene glycol) hydrogels. Biomacromolecules 2002;3:1038-1047.

8. Park JY, Kim JS, Nam YS. Mussel-inspired modification of dextran for protein-resistant coatings of titanium oxide. Carbohydr Polym 2013;97:753-757.

9. Lee C, Shin J, Lee JS, Byun E, Ryu JH, Um SH, et al. Bioinspired, calcium-free alginate hydrogels with tunable physical and mechanical properties and improved biocompatibility. Biomacromolecules 2013;14:2004-2013.

10. Hong SH, Shin M, Lee J, Ryu JH, Lee S, Yang JW, et al. STAPLE: stable alginate gel prepared by linkage exchange from ionic to covalent bonds. Adv Healthc Mater 2016;5:75-79.

11. You I, Kang SM, Byun Y, Lee H. Enhancement of blood compatibility of poly(urethane) substrates by mussel-inspired adhesive heparin coating. Bioconjug Chem 2011;22:1264-1269.

12. Lee Y, Lee SH, Kim JS, Maruyama A, Chen X, Park TG. Controlled synthesis of PEI-coated gold nanoparticles using reductive catechol chemistry for siRNA delivery. J Control Release 2011;155:3-10.

13. Ryu JH, Lee Y, Kong WH, Kim TG, Park TG, Lee H. Catechol-functionalized chitosan/pluronic hydrogels for tissue adhesives and hemostatic materials. Biomacromolecules 2011;12:2653-2659.

14. Kim YM, Kim CH, Park MR, Song SC. Development of an injectable dopamine-conjugated poly(organophophazene) hydrogel for hemostasis. Bull Korean Chem Soc 2016;37:372-377.

15. Mehdizadeh M, Weng H, Gyawali D, Tang L, Yang J. Injectable citrate-based mussel-inspired tissue bioadhesives with high wet strength for sutureless wound closure. Biomaterials 2012;33:7972-
7983.

16. Fan C, Fu J, Zhu W, Wang DA. A mussel-inspired double-crosslinked tissue adhesive intended for internal medical use. Acta Biomater 2016;33:51-63.

17. Kim K, Ryu JH, Koh MY, Yun SP, Kim S, Park JP, et al. Coagulopathy-independent, bioinspired hemostatic materials: a full research story from preclinical models to a human clinical trial. Sci Adv 2021;7:eabc9992.

18. Burks S, Spotnitz W. Safety and usability of hemostats, sealants, and adhesives. AORN J 2014;100:160-176.

19. Emilia M, Luca S, Francesca B, Luca B, Paolo S, Giuseppe F, et al. Topical hemostatic agents in surgical practice. Transfus Apher Sci 2011;45:305-311.

20. Mehdizadeh M, Yang J. Design strategies and applications of tissue bioadhesives. Macromol Biosci 2013;13:271-288.

21. Samudrala S. Topical hemostatic agents in surgery: a surgeon's perspective. AORN J 2008;88:S2-S11.

22. Albes JM, Krettek C, Hausen B, Rohde R, Haverich A, Borst HG. Biophysical properties of the gelatin-resorcin-formaldehyde/glutaraldehyde adhesive. Ann Thorac Surg 1993;56:910-915.

23. Brennan M. Fibrin glue. Blood Rev 1991;5:240-244.

24. Tomizawa Y. Clinical benefits and risk analysis of topical hemostats: a review. J Artif Organs 2005;8:137-142.

25. Lewis KM, Spazierer D, Urban MD, Lin L, Redl H, Goppelt A. Comparison of regenerated and non-regenerated oxidized cellulose hemostatic agents. Eur Surg 2013;45:213-220.

26. Schwartz M, Madariaga J, Hirose R, Shaver TR, Sher L, Chari R, et al. Comparison of a new fibrin sealant with standard topical hemostatic agents. Arch Surg 2004;139:1148-1154.

27. Frilling A, Stavrou GA, Mischinger HJ, de Hemptinne B, Rokkjaer M, Klempnauer J, et al. Effectiveness of a new carrier-bound fibrin sealant versus argon beamer as haemostatic agent during liver resection: a randomised prospective trial. Langenbecks Arch Surg 2005;390:114120. 